Originalveröffentlichung in: Beyer, Andreas ; Simon, Ralf ; Stierli, Martino (Hrsgg.): Zwischen Architektur und literarischer Imagination. München u.a. 2013, S. 145-167 (eikones)

\title{
"Ich aber bin entstellt von Ähnlichkeit mit allem, was hier um mich ist." Das Motiv des architektonischen Selbstporträts in Literatur und Architektur
}

Matthias Noell

"Die Gabe, Ähnlichkeiten zu erkennen, ist ja nichts als ein schwaches Überbleibsel des alten Zwanges, ähnlich zu werden und sich zu verhalten. Den aber übten Worte auf mich aus. Nicht solche, die mich Mustern der Gesittung sondern Wohnungen, Möbeln, Kleidern ähnlich machten. Nur meinem eignen Bilde nie. Und darum wurde ich so ratlos, wenn man Ähnlichkeit mit mir selbst von mir verlangte. Das war beim Photographen. [...] Ich aber bin entstellt von Ähnlichkeit mit allem, was hier um mich ist. ${ }^{1}$

\section{Verähnlichungen}

In seinem Text Mummerehlen aus der Berliner Kindheit um 1900 äußerte sich Walter Benjamin zu der grundlegenden Verähnlichung seines Ich mit den ihn umgebenden Dingen, sogar mit ganzen Wohnungen und Interieurs. Es fiel Benjamin leichter, sich in dem chinesischen Porzellan wiederzuerkennen, das er als Kind des häufigeren abzeichnete, als auf Fotografien, die man von ihm anfertigte. Seine kindliche Erfahrung sah Benjamin in der Geschichte der Muhme Rehlen bestätigt, in der ein Maler in seinem Gemälde verschwindet: 
»Sie [die Erzählung] stammt aus China und erzählt von einem alten Maler, der den Freunden sein neuestes Bild zu sehen gab. Ein Park war darauf dargestellt, ein schmaler Weg am Wasser und durch einen Baumschlag hin, der lief von einer kleinen Türe aus, die hinten in dem Häuschen Einlaß bot. Wie sich die Freunde aber nach dem Maler umsahen, war der fort und in dem Bild. Da wandelte er auf dem schmalen Weg zur Tür, stand vor ihr still, kehrte sich um, lächelte und verschwand in ihrem Spalt. So war auch ich bei meinen Näpfen und den Pinseln auf einmal ins Bild entstellt. Ich ähnelte dem Porzellan, in das ich mit einer Farbwolke Einzug hielt. « ${ }^{2}$

Die beiden Bestandteile der Benjaminschen Verähnlichung, die Angleichung an das den Erzähler Umgebende und das Verschmelzen mit der selbst erschaffenen Kunst, sind gleichermaßen Topoi in der Literatur wie in der Bildenden Kunst, sie sind aber auch in der Architektur und Architekturtheorie nachweisbar. Die Stellvertretung, manchmal sogar Gleichsetzung von Menschen mit ihren architektonischen Gehäusen, ist ein bislang jedoch wenig beachtetes Phänomen in der Architekturgeschichte der letzten 200 Jahre. Der vorliegende Beitrag stellt erstmals eine Auswahl solcher Beispiele zusammen; er folgt Ernst Kris' und Otto Kurz' Idee des "geschichtlichen Versuchs" und kann als eine Ergänzung ihres Buchs Die Legende des Künstlers verstanden werden. ${ }^{3}$

\section{Haus und Mensch, eine neue Einheit}

Anthropomorphe Interpretationen von Architektur können, zumindest wenn sie von konkreten Personen handeln, die mit Bauwerken in Analogie gesetzt werden, eine Erinnerungsfunktion beinhalten. Im Roman Bruges-la-Morte von Georges Rodenbach entsteht in der Wahrnehmung des Protagonisten Hugues Viane eine Einheit seiner verstorbenen Frau mit der stoten Stadt Brügge: »Brügge war seine Tote. Und seine Tote war Brügge. Alles vereinigte sich im gleichen Schicksal. ${ }^{4}$ Auch in Valérys Erzählung Eupalinos ou l'architecte steht steinerne Architektur sinnbildlich für eine Verstorbene. Die Analogiebildung zwischen dem menschlichen Körper eines korinthischen Mädchens und einem kleinen, eleganten Hermes-Tempel ist sicherlich auch von Ovids Metamorphosen angeregt, wobei Valéry nicht von einer Verwandlung eines Menschen in andere Körper oder in ein anderes Material berichtet, sondern der Architektur die Funktion eines abstrakten Porträts zuweist: 
»O süße Verwandlung! Dieser zarte Tempel, niemand ahnt es, ist das mathematische Bildnis eines Mädchens von Korinth, das ich glücklich geliebt habe. Er wiederholt getreu die besondren Verhältnisse ihres Körpers. Er lebt für mich! Er gibt mir zurück, was ich ihm gegeben habe ...-Deshalb also ist er von so unerklärlicher Anmut, erwiderte ich ihm. Man fühlt wirklich in ihm die Gegenwart einer Person, die erste Blüte einer Frau, die Harmonie eines entzückenden Wesens; er erweckt ungefähr eine Erinnerung, die es nicht bis zu ihrem Umriß bringt; und dieser Anfang eines Bildnisses, das du in seiner Vollendung besitzt, genügt, die Seele zu locken und zugleich zu bestürzen. $\varkappa^{5}$

Grundlagen für die Parallelisierung von menschlichem Charakter und Architektur finden sich bereits im 18. und frühen 19. Jahrhundert, sei es nun in architekturtheoretischen Schriften oder auch in der Literatur. Alain-René Lesages Le diable boiteux (1707), Nicolas Le Camus de Mézières' Schrift Le génie de l'architecture ou l'analogie de cet art avec nos sensations (1780), oder der anonym gebliebene Autor der Untersuchungen über den Charakter der Gebäude (1785) wären hierfür geeignete Belege. ${ }^{6}$ Insbesondere Letzterer mit seiner Idee, jedes Gebäude sei »ein körperliches Symbol von den Bedürfnissen des Menschen und seinem Zustande«, ${ }^{7}$ fand nur wenig später einen berühmten Nachfolger. Johann Wolfgang von Goethe beschrieb 1795 die Übereinstimmung des menschlichen Charakters und des eigenen Wohnhauses in einem Brief an Heinrich Meyer, "des Menschen Wohnung ist sein halbes Leben«, und an Karl Ludwig von Knebel: "Der Mensch ist seinem Wohnort so nahe verwandt, daß die Betrachtung über diesen auch uns über den Bewohner aufklären muß. ${ }^{8}$ In Wilhelm Meisters Wanderjahre führte Goethe dieses Bild weiter aus und wies der Architektur sogar die Rolle einer Protagonistin zu:

„Gewiß, Ihr bewundert die Übereinstimmung dieses Gebäudes mit seinen Bewohnern, die Ihr gestern kennen lerntet. Sie ist aber vielleicht noch sonderbarer, als man vermuten sollte: das Gebäude hat eigentlich die Bewohner gemacht. Denn wenn das Leblose lebendig ist, so kann es auch wohl Lebendiges hervorbringen. ${ }^{9}$

Auch der englische Antiquar und Verleger John Britton sah 1827 im Haus und im privaten Wohnumfeld einen direkten 
Ausdruck des menschlichen Charakters-und richtete sich direkt gegen den Umweg über phrenologische Studien in der Folge Franz Joseph Galls, die die unterschiedlichen Hirnareale ja ihrerseits als Kammern und Zimmer illustrierten:

"so does the house of the Architect, the gallery of the Painter, and the library of the Author, exhibit some prominent characteristic trait of its respective owner. Instead of vainly attempting to prognosticate the ruling passion and character by phrenological bumps, or craniological organs, we shall find a better and surer criterion of judging man, by referring to his domestic habits and associations. ${ }^{10}$

Allen gemeinsam war die Überzeugung, dass »des Wohners Sinn im Hause« erkannt werden könne, wie es Wilhelm von Humboldt in einem Sonett formulierte-möglicherweise sogar noch nach dessen Tod. ${ }^{11}$

Die charakterliche Gleichsetzung des Schöpfers mit seinem architektonischen Werk scheint in der Zeit um 1900, als es den Reformkünstlern explizit um die Einheit von Leben und Werk ging, verstärkt aufgegriffen worden zu sein. Heinrich Vogeler schrieb beispielsweise an den befreundeten Schriftsteller Hans Bethge, der Barkenhoff, sein nach eigenen Plänen umgebautes Worpsweder Bauernhaus, sei »ein Stück von mir", "meine ganze Seele wohnt hier «. ${ }^{12}$ Karl Schefflers Kommentar zu Peter Behrens' eigenem Wohnhaus auf der Darmstädter Mathildenhöhe, in dem er die Entstehung der Architektur aus dem Charakter seines Entwerfers, und damit eine unverfälschte, ehrliche und daher auch moderne Haltung postulierte, kann als ein nüchternes Beispiel dieser Lesart des Künstleroder Architektenwohnhauses gelten: „Das Haus zeugt in jeder Form von der Art seines Erbauers. ${ }^{13}$

Bei Marcel Proust avancierten Architektur und Interieur für den sensiblen Betrachter und Besucher sogar zu einem Platzhalter ihres abwesenden Bewohners, werden also wiederum zum Anlass eines Erinnerungsprozesses. Nicht nur werden hier der Charakter des Zimmers und seiner Einrichtung in Beziehung zu seinem Bewohner gesetzt, die Innenarchitektur zeugt nicht mehr nur von der >Art ‘ ihres Bewohners, dieser wird jetzt sogar darin vergegenwärtigt, nahezu aufgelöst. In der Recherche-den ersten Band sollte Walter Benjamin gemeinsam mit Franz Hessel in den 1920er Jahren übersetzen - thematisierte Proust diese Repräsentation eines Menschen durch seine persönlichen Gegenstände und Räume: 
»Saint-Loup schien darin [in seinem Zimmer] fast leiblich gegenwärtig zu sein dank den Büchern, die auf seinem Arbeitstisch lagen neben den Photographien, unter denen ich die meine und die von Madame de Guermantes erkannte, und dank dem Feuer, das sich jetzt an den Kamin gewöhnt hatte ${ }^{14}{ }^{14}$

Möglich wird hier die vermeintliche Präsenz der abwesenden Person durch die Gegenstände und den Raum selbst, vor allem aber durch die Einfühlung des Erzählers in die Gefühlswelt des Abwesenden, die in Fotografie und Büchern nachvollzogen wird. In seinem Zimmer ist der abwesende Bewohner in der erwarteten Rückkehr und in seinen Gegenständen anwesend-unter anderem in der Form einer Spiegelung des Erzählers in zwei Porträtfotografien von sich selbst und seiner insgeheim Geliebten. Bei Proust ist nicht mehr des »Wohners Sinn" im Haus zu entdecken, es ist der Bewohner selbst.

Jenseits dieser literarischen Imaginationen bediente sich auch die Architekturkritik vergleichbarer Bilder. Der Schriftsteller Fritz von Ostini hatte die Analogie von Werk und Architekt, vielleicht ausgehend von solchen Formulierungen wie der des zitierten Karl Scheffler, wenn nicht geprägt, so in jedem Fall ebenso anschaulich und eingängig wie weitschweifig eingesetzt, als er 1909 einen Artikel zu Franz von Stucks Atelierwohnhaus in München für die Innen-Dekoration, dem einflussreichen Magazin des Darmstädter Verlegers Alexander Koch, verfasste:

"Das Haus ist der Mensch! Immer vorausgesetzt, daß der Mensch sich dies Haus selber gebaut und nicht beim Baumeister bestellt hat, wie einen Rock beim Schneider. Und wenn ein Künstler sich sein Heim baut, so spiegelt sich sein wahres Wesen darin oft viel klarer wieder, als in einem einzelnen Werke. Weil in seinem Hause nicht nur sein Können und sein künstlerischer Geschmack zum Ausdruck kommen, sondern auch seine Stellung zum Leben, sein menschliches Temperament, seine ganze Physis. Ein Künstler, der Raumkunst treibt für einen Andern, für fremde Bedürfnisse, arbeitet ganz anders, als der, der sich selbst das Gehäuse anmißt, in dem er leben und einst sterben will-und den vielleicht auch noch die Absicht leitet, in diesem Bau sein stärkstes und kennzeichnendstes Kunstwerk zu schaffen. So steht auch Franz v. Stuck zu seinem Haus, das er sich im 
Jahr 1898, als ein Fünfunddreißigjähriger auf der GasteigHöhe in München errichtet hat: festumschlossen und einheitlich, stark, klar und gradlinig wie seine ganze Persönlichkeit ist auch sein Heim. Breit trotzig und selbstbewußt, anders als das Andere ringsumher, umweht vom Hauch der Antike und doch modern, monumental, ja prunkvoll, aber ohne theatralische Gebärde-das ist das Haus, das ist der Mann, das ist der Maler! [...] Dies Haus ist der Mann! « ${ }^{15}$

Noch vierzig Jahre später lautete Friedrich Kieslers Ausspruch nahezu wortgleich: "Larchitecte est sa maison. La maison est l'architecte. ${ }^{16}$

Kuno Graf von Hardenberg griff in dem 1926 erschienenen Buch zum eigenen Wohnhaus Alexander Kochs auf jene Idee der Einheit des Besitzers mit seinem Haus zurück. Explizit wird hier das Arbeitszimmer [Abb.1] als >Matritze des Wesens seines Besitzers bezeichnet, es sei die

"Verkörperung seiner eigensten Seelenbedürfnisse und seines eigensten Wesens! Es spricht dem, der in Räumen lesen kann, wie in Büchern oder Herzen, von allem, was der Herr ist, will und kann, tut, wünscht, genießt, - es ist der Vollausdruck der Persönlichkeit des Bewohners und das ist das Köstlichste an ihm, ist mehr als Luxus und Prunk aller Welt. ${ }^{17}$

Diese sogenannte Verkörperung war noch im selben Jahr Leitthema in einem weiteren Heft der Innen-Dekoration:

"Man sagte einmal, die Kleidung sei ein erweiterter körperlicher Wesens-Ausdruck des Menschen; in gleicher Weise ist es sicher auch das Heim! [...] Gemeinhin ist es so, daß wir die Persönlichkeit eines Menschen bewußt in seiner Wohnung gespiegelt finden, und das Heim stellt fast in allen Fällen seinem Gestalter einen spsychologischen Steckbrief ‘ aus. Nicht selten macht es uns sogar Hauptmerkmale eines Charakters deutlicher, als wir sie im gewöhnlichen Umgang gewahren. $\aleph^{18}$

Ein weiterer Artikel desselben Hefts thematisierte die Verbindungen zwischen Seele und Wohnung:

"Unser seelisches System ist wie eine `Zweizimmer-Wohnung ‘ Zunächst ein nach der Straße hinaus gelegenes, einfenstriges, 
kleines, freundlich erhelltes Vorderzimmer (unser bewußtes Ich), das ausgestattet ist mit einer sehr empfindlichen Tapete (nämlich der Moral). Daran anstoßend eine ungleich höhere, meist völlig lichtlose Kammer (unser unbewußtes Ich). ${ }^{19}$

Dass eine solche Übereinstimmung von Entwerfer und Gebäude auch Züge ins Pathologische aufweisen kann, führte Thomas Bernhard 1975 in seinem Roman Korrektur aus. Die Grenzen zwischen der beschützenden Hülle des Menschen und dem Ich des Architekten beginnen hier in der Wahrnehmung des Betrachters bedrohlich zu verschwimmen:

»Vor allem hatte ich sofort die Vergleichsmöglichkeit, indem ich den Höller anschaute und sein Haus anschaute und den Höller studierte und sein Haus studierte und das Charakteristische des Höller war auch das Charakteristische des Höllerhauses, wie das Innere des Höller auch das Innere des Höllerhauses, dadurch, daß ich das Höllerhaus studierte, hatte ich plötzlich Einblick in den Höller, umgekehrt, indem ich den Höller studierte, Einblick in das Höllerhaus, das eine war gleichzeitig die Erklärung auch des anderen. Ich hätte ohne weiteres sagen können, so Roithamer, das Innere des Höller ist das Innere seines Hauses. « ${ }^{20}$

Auch die Avantgarde der 1920er Jahre bediente sich der metaphorischen oder vergleichenden Betrachtung von Haus und Mensch, der Gleichsetzung von Individuum und Architektur. In einer Architekturkritik Theo van Doesburgs anlässlich der Werkbundausstellung auf dem Stuttgarter Weißenhof kommt dieser, an die Untersuchungen über den Charakter der Gebäude erinnernde Aspekt deutlich zum Tragen: »Häuser sind wie Menschen. An ihren Gesichtszügen, ihrer Haltung, dem Gang, der Kleidung, kurz: an ihrer ’Oberfläche` läßt sich ihr Denken, ihr Innenleben ablesen. ${ }^{21}$ Bruno Taut schrieb über die Wohnung und ihren Bewohner, sie sei »der unmittelbarste und grausamste Spiegel jedes einzelnen Menschen «, ${ }^{22}$ was seinen Rezensenten Hans Josef Zechlin wiederum zu einer unvermittelten Analogie von Baumonografie und Autobiografie animierte:

"Wir wissen, wieviel Eitelkeiten wir in Abzug bringen müssen, um eines Schriftstellers Autobiographie recht zu genießen-in diesem Bekenntnis eines Architekten vermissen wir angenehm das so leicht zu begreifende Schielen nach 


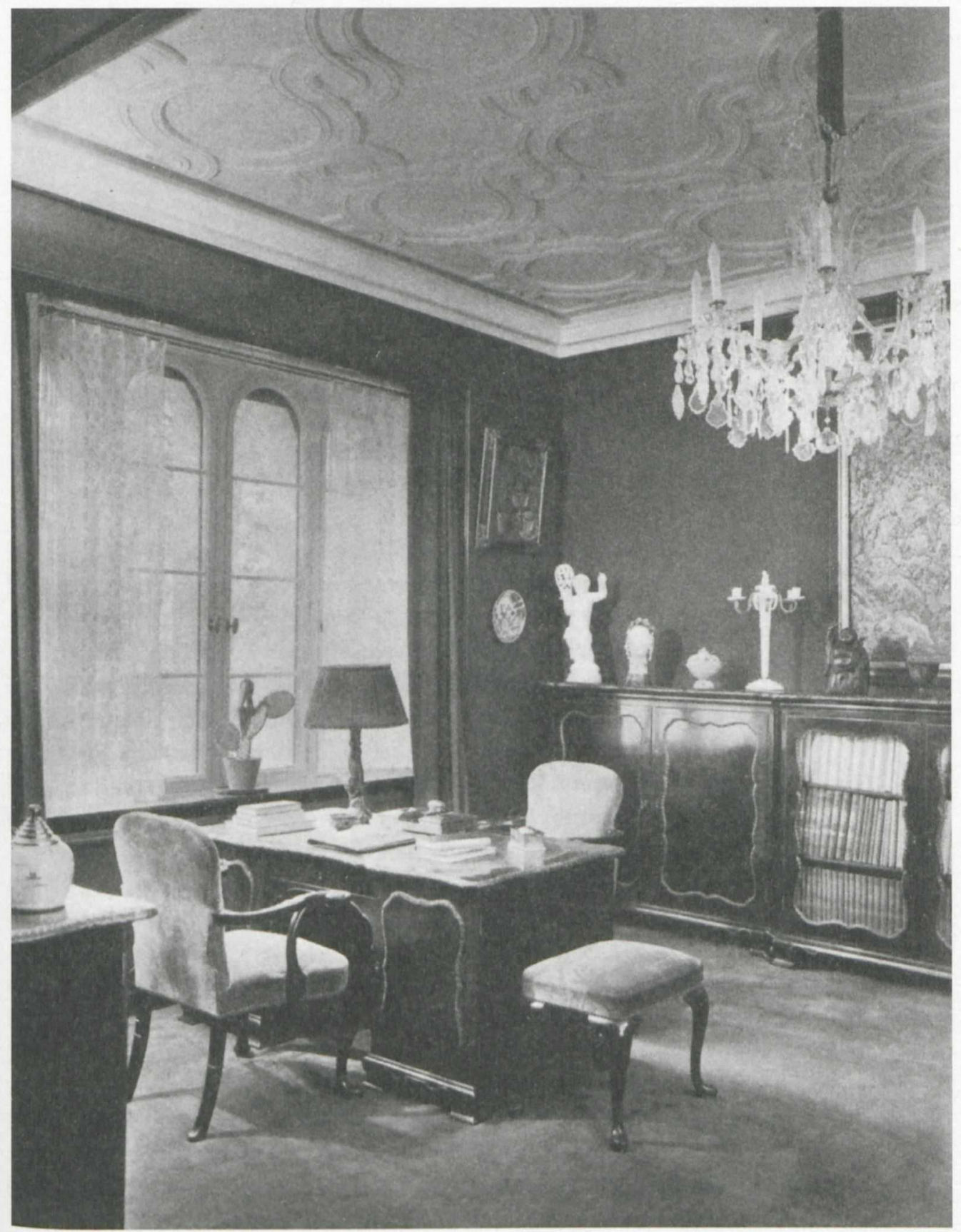

1 Hugo Schmölz, Fensterplatz mit Schreibtisch im Herrenzimmer, $1925 / 26$.

"lch aber bin entstellt von Ähnlichkeit mit allem, was hier um mich ist.، 


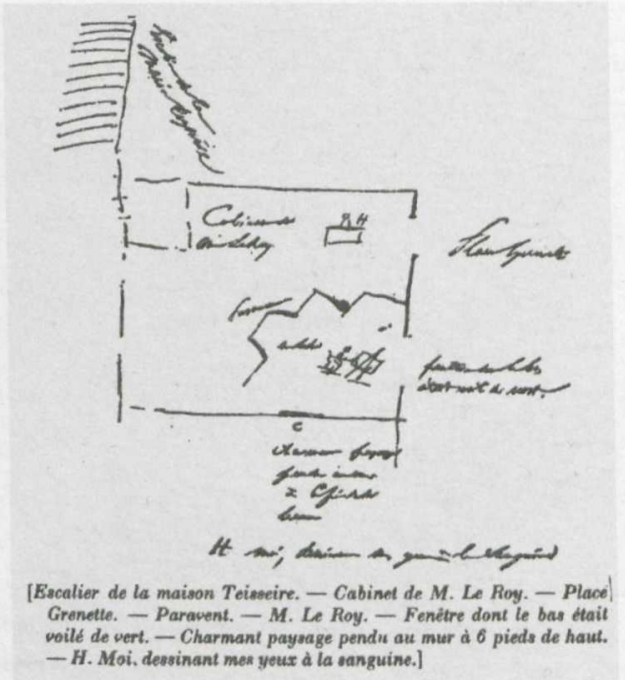

2 Stendhal, Cabinet de M. Le Roy, um 1835/36. dem kommenden Bauherrn. [...] So gibt Bruno Taut in diesem Buch ein menschliches Bekenntnis zu sich selbst. $^{23}$

Autobiografie und eigenes Wohnhaus werden hier explizit miteinander in Verbindung gebracht. Der Schluss, das eigene Wohnhaus in Analogie zur literarischen Äußerung als ein gebautes Selbstporträt zu interpretieren, ist also in Zechlins Rezension über die Gleichsetzung von Haus und Mensch hinaus ebenfalls enthalten. ${ }^{24}$

\section{Repräsentationen des Inneren: \\ Der Raum als autobiographisches Werk}

Schon in einem Brief Goethes an die Gräfin Stolberg von 1775, der er eine Zeichnung seines Frankfurter Zimmers beilegte, vor allem aber in Stendhals Vie de Henri Brulard findet sich in Ansätzen eine Verbindung von literarischer Autobiografie und architektonischem Innenraum. Lakonisch verkürzte Beschreibungen ergänzte Stendhal durch genaue, wenn auch laienhaft wirkende Skizzen, die, so Béatrice Didier, Erinnerungshilfe, Konzeption und Illustration gleichermaßen seien. ${ }^{25}$ Die solcherart umschriebenen Innenräume-wie zum Beispiel das Atelier des Malers Le Roy [Abb.2] -finden sich an den zentralen Stellen des Textes und betten Stendhals Wahrnehmung und sein künstlerisches Ich in eine Reihe von Personen- und Ortsbezügen ein. ${ }^{26}$

Es liegt nahe zu fragen, inwieweit solche literarischen und theoretischen Sichtweisen auf Interieur und Architektur Parallelen 
in deren bildlicher Darstellung haben. Insbesondere im Fall gemalter und fotografierter Künstlerateliers ist sich die Forschung seit langem darüber einig, dass diese Gattung im Regelfall eine Vergewisserung des künstlerischen Ich beinhaltet. Eigene Atelierdarstellungen transportieren häufig genau jene Stimmungswerte, die auch den Selbstporträts der Künstler zu eigen sind. Dabei ist es nicht einmal von Bedeutung, ob das Atelier nun mit oder ohne den Künstler gezeigt wird. Der Betrachter sieht den Raum des Künstlers mit dessen Augen, was aus der Atelierdarstellung gleichermaBen eine programmatische Erläuterung und Einweihung in dessen kunsttheoretische Position und Weltsicht werden lässt. Die Atelierdarstellung-und hier ist seit dem letzten Drittel des 19. Jahrhunderts zunehmend die Fotografie beteiligt-beinhaltet als »Dokument thematisierter Künstlerpsychologie«, wie es Werner Hofmann ausdrückte, daher immer auch die Möglichkeit einer Interpretation als Porträt oder Selbstporträt des Künstlers. ${ }^{27}$

Schon Ende des 19. Jahrhunderts hatten zahlreiche Künstler, zu nennen wären beispielsweise Edgar Degas oder Pierre Bonnard, in der Fotografie ein geeignetes Medium für experimentelle Porträts und Selbstporträts im Atelier entdeckt. ${ }^{28}$ Die Fotografie konnte innovative Gestaltung mit einem Maximum an Authentizität verbinden, ohne dabei auf die Inszenierung des auratisch aufgeladenen Schaffensorts im Bild verzichten zu müssen. Gleiches gilt sehr häufig auch für die Atelierdarstellungen, die befreundete Fotografen anfertigten. Die zahlreichen Fotografien der Ateliers 
von Piet Mondrian sind hierfür beredte Beispiele. Sie richten in den allermeisten Fällen den Blick auf die Staffelei oder direkt auf mehrere Gemälde an der Wand. Dabei ging es den verschiedenen Fotografen, etwa Paul Delbo, Charles Karsten, Fritz Glarner, Arnold Newman oder Harry Holtman, meistens darum, die Gestaltung des Ateliers - ganz im Sinne Mondrians-in Form eines großen Raumgemäldes abzubilden und zu inszenieren. ${ }^{29}$ Daher ähneln die fotografischen Aufnahmen in ihrer Komposition nahezu von selbst den abgebildeten Gemälden. Sie visualisieren, wie die gemalten Atelierdarstellungen selbst meist auch, »ein Stück Kunstlehre «. ${ }^{30}$ Der Fotograf André Kertész ging darin sogar noch ein Stück weiter. Er versuchte nicht nur, das bildnerische Schaffen Mondrians zusammen mit dem Raum des Ateliers in einer Fotografie zu verknüpfen, sondern übertrug die Präsenz des Künstlers und seines ebenfalls anwesenden Freundes und Kollegen Michel Seuphor-ähnlich wie in der zitierten Passage Prousts-auf einige charakteristische persönliche Gegenstände. Die 1926 entstandene Fotografie des Eingangsbereichs mit Garderobe, Mantel, Hut (von Michel Seuphor) und 


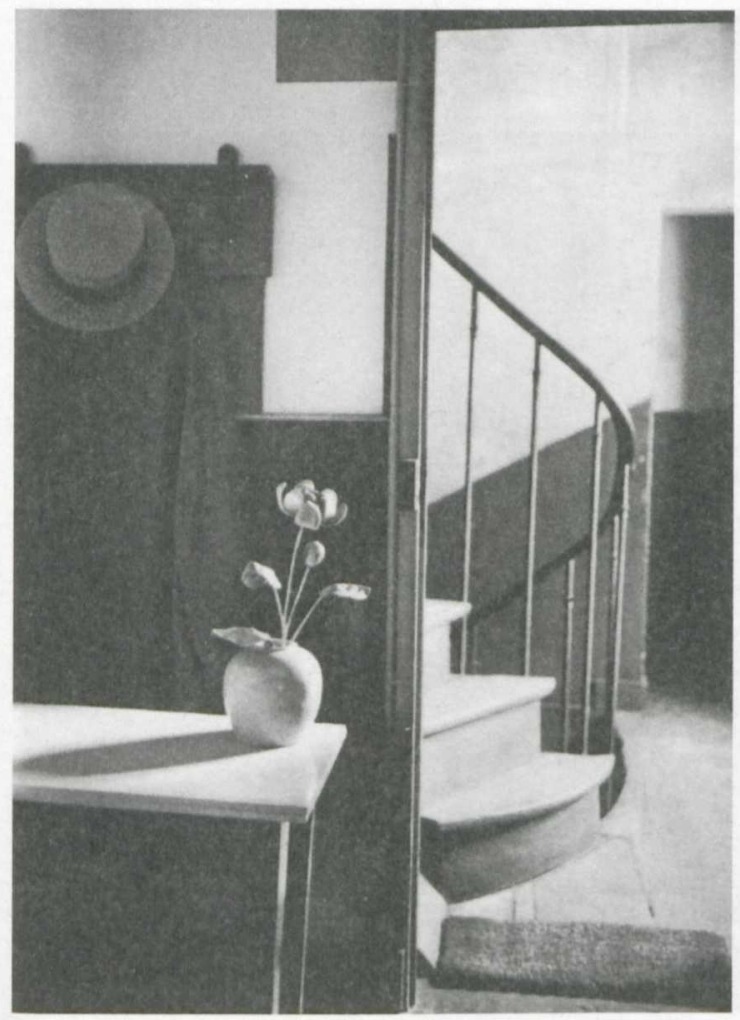

der künstlichen Tulpe als kunsttheoretisches Zeichen überführt in der Anordnung der Objekte im architektonischen Raum die flächigen Abstraktionen Mondrians in die Grauwerte der Fotografie [Abb. 3]. ${ }^{31}$ So tritt uns in der Fotografie von Kertész eine dreifache Repräsentation des im Bild abwesenden Künstlers entgegen-über sein dargestelltes Atelier, über die abgelichteten persönlichen $\mathrm{Ge}$ genstände, auch die seines Besuchs, und über die abstrakte Flächenkomposition der Fotografie selbst. Daher ist auch die fotografische Darstellung zweier Brillen und der Pfeife Mondrians auf einem Tisch, wie sie Kertész ebenfalls 1926 anfertigte, als solches sublimiertes Künstlerporträt anzusehen, zumal bereits Gustave Courbet um die Mitte des 19. Jahrhunderts diverse Darstellungen von Gegenständen als Selbstporträt bezeichnet hatte, unter anderem sein Selbstporträt in Gestalt einer Pfeife von 1858. Und auch hier, in der aufsichtig und übereck fotografierten, hellen, Tischplatte vor dem nahezu schwarzen Hintergrund, übersetzte und interpretierte Kertész die abstrakten Kompositionen Mondrians. Hut, Mantel, Brille und Zigaretten, die Lucien Hervé in den Innenraumfotografien der

3 André Kertész, Atelier Piet Mondrians in der Rue de Départ in Paris, 1926. 
Villa Savoye in Poissy in Szene setzte, können demnach ebenso als Porträts ihres Besitzers Le Corbusier verstanden werden. ${ }^{32}$ Die beschriebene Arbeitsweise prägt aber auch weitere Atelierfotografien Kertész'. Michel Frizot äußerte bezüglich einer Aufnahme des Ateliers von Fernand Léger, Kertész sei es darum gegangen, mein Ambiente einzufangen und dabei eine Art verschobenes Porträt des Malers zu erstellen, wobei die Syntax von Gegenständen ohne erkennbaren Zusammenhang die Vorlieben, Gewohnheiten und eine bezeichnende Raumgestaltung widerspiegelt. [...] Die Ausarbeitung des Arrangements erinnert ihrerseits an Légers Bilder «. ${ }^{33}$

Weitere Beispiele für diese programmatische Form künstlerischer Inszenierung sind in den Fotografien der Meisterhäuser in Dessau zu finden, die Lucia Moholy für die Publikation Bauhausbauten Dessau anfertigte. ${ }^{34}$ Im leergefegten Atelier László Moholy-Nagys steht, stellvertretend für den abwesenden Bewohner ein zwar persönlicher, aber gleichermaßen unpersönlich-industrieller Gegenstand: 


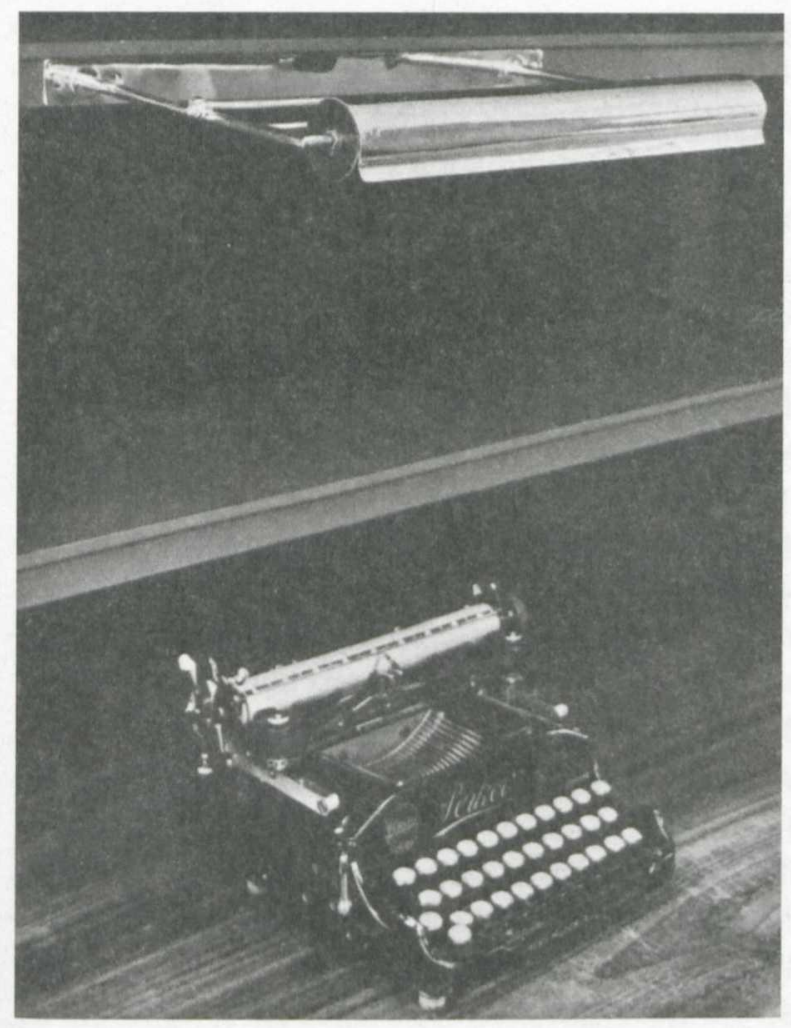

Die Schreibmaschine wird zum Sinnbild für den modernen, denkenden und schreibenden Künstler, und zugleich für die Präzision der modernen, maschinellen Welt der seriellen Produktion. ${ }^{35}$ Auf diesen Fotografien - auch die Schreibmaschine von Walter Gropius [Abb. 4] ist hierfür ein Beleg-konnte ein simples technisches Gerät einerseits stellvertretend für den modernen Künstler stehen und somit die Rolle eines Porträts einnehmen. Andererseits konnte es auch für das Haus selbst stehen: »Aber stolz kann man auf ein Haus sein«, schrieb Le Corbusier, »das praktisch wie die eigene Schreibmaschine ist. ${ }^{36}$

\section{Steinerne Porträts}

Haus und Atelier oder sogar nur Teile des Ateliers können also, je nach Zusammenhang und Verwendung, dem Künstler anverwandelt und verähnlicht, und damit stellvertretend für ihn eingesetzt werden. Der ins Bild gesetzte architektonische Raum überschreitet in diesen Fällen seine Aufgabe als Bühne des menschlichen Handelns und wird stattdessen zum Abbild eines abwesenden Protagonisten.

4 Lucia Moholy, Wohnungen der Bauhausmeister. Schreibtischklapplampe am Doppelschreibtisch des Einzelhauses Gropius, 1926. 
Derselbe Sachverhalt gilt nicht nur für das gemalte oder fotografierte Abbild des Ateliers, sondern auch für das Haus selbst. Diese zunächst etwas eigenwillig wirkende Funktion von Architektur-man muss auch diese Form der Repräsentation wohl als Funktion begreifen-findet sich in der Verbindung zahlreicher Orte, Architekturen und Protagonisten. Paul Valéry hatte seinen Baumeister Eupalinos diese Konstruktion des Künstler-Ich im eigenen architektonischen Werk mit ironischer Brechung resümieren lassen: »Indem ich baute, warf er lächelnd hin, habe ich mich, glaube ich, selbst erbaut. « ${ }^{37}$

Dass nicht nur das Abbild des Interieurs, sondern auch die Architektur selbst als "gebautes Selbstporträt" gelesen werden kann, haben Christine Hoh-Slodczyk oder François Chaslin schon vor etlichen Jahren bemerkt. ${ }^{38}$ Und auch hierfür gibt es literarische Belege: So beschrieb Curzio Malaparte sein gesamtes Haus als unbewusst entstandenes Abbild seiner selbst. Ganz konkret äußerte er sich zur Übereinstimmung zwischen seinem Haus, dessen Adresse auf Capri er auf seinem Briefkopf mit »Casa come me« bezeichnete, und seiner Person:

»An dem Tag, an dem ich mit dem Bau des Hauses begann, ahnte ich nicht, daß ich ein Bildnis meiner selbst entwerfen würde. Noch dazu ein besseres als ich bis heute jemals in meinen Büchern gezeichnet habe. [...] ich hatte mich noch nie so sehr gezeigt, wie ich wirklich bin, wie in dem Versuch, ein Haus zu bauen. ${ }^{39}$

"Traurig, hart und streng« sei sein Haus geworden, so Malaparte, "ein steinernes Porträt«. ${ }^{40}$ Malaparte steht jedoch mit dieser durchaus exaltierten Haltung nicht allein. Der Architekt Tomaso Buzzi übernahm für seine Idealstadt im ehemaligen Franziskanerkloster La Scarzuola, das er 1956 erwarb, die Idee des steinernen Porträts-vermutlich direkt von Malaparte. ${ }^{41}$ Die Parallelisierung von eigenem, persönlichem und architektonischem Charakter kann aber etwa zeitgleich auch in einem Kommentar des schwedischen Architekten und Herausgebers der Architekturzeitschrift Byggmästaren, Leif Reinius, nachgelesen werden, der in einer Einführung zu neuen schwedischen Villenbauten das 1945/46 erbaute Haus seines Kollegen Sven Ivar Lind vorstellte und folgendermaßen beschrieb: „Das Ganze ist ein Selbstporträt von Lind; geprägt von Verfeinerung, Blässe und von klassischer Klarheit. ${ }^{42}$ In nur wenigen Jahren hatte sich der bei Karl Scheffler zu lesende, zurückhaltende Vergleich von Haus und Mensch zu einem literarisch-architektonischen Selbstporträt 
verändert und als Topos etabliert, der auch von der kunsthistorischen Forschung aufgegriffen wurde. Alfred Jericke, der Weimarer Kunsthistoriker und `Biografı des Goetheschen Hauses am Frauenplan, formulierte dies sicher in bewusster Verwendung des oben zitierten Goetheschen Motives: "Beinahe wie in einem Buche, einem autobiographischen Werke, sollten wir darin [in seinem Haus] lesen « ${ }^{43}$ Gustav Friedrich Hartlaub verglich Porträt- und Gebäudefotografien von Henry van de Velde, Heinrich Tessenow, Hans Poelzig, Wilhelm Kreis und Walter Gropius, und schloss aus der Betrachtung:

"Es zeigte sich, daß meßbar statische wie auch funktionelle Eigenschaften, dazu der undefinierbare Gesichtsausdruck (wie das alles die Photographie an einem Künstler feststellt) eigentümlich transponiert auch und gerade in den baulichen Schöpfungen wiederkehren! « ${ }^{44}$

Dass Architektur und ihr Abbild schließlich auch Porträt eines Amtes sein können, bewies der Porträtfotograf Paul Swiridoff mit seinem Bildband Der Bungalow. Als Stellvertreterporträt des nur auf Zeit amtierenden deutschen Bundeskanzlers wird hier nicht nur die Architektur des Hauses, sondern auch die demokratische Staatsform als solche präsentiert. Der Bungalow sei »Ausdruck politischer Gesinnung «, fasste Erich Steingräber am Ende des Buchs zusammen. ${ }^{45}$

Was aber passiert nun, wenn ein Künstler, Literat oder Architekt sich selbst in sein Haus einschreibt, in seinem Werk verschwindet, wie der Maler in Benjamins Erzählung, und damit seine Anwesenheit möglicherweise oder sogar notwendigerweise überflüssig macht? Noch zu Lebzeiten, so könnte man angesichts der erhaltenen Künstlermuseen überspitzt formulieren, ersetzt er sich durch die Erinnerung an sich und errichtet sich selbst einen vorzeitigen Kenotaph. Ein Beispiel aus der jüngeren Vergangenheit ist bestens geeignet, diesen Konflikt zu illustrieren, und soll daher diesen Überblick über das Thema des architektonischen Selbstporträts beschließen. Der französische Künstler Jean-Pierre Raynaud fasste in den 1960er Jahren den Entschluss, sich und seiner Familie ein Haus zu errichten. Es wandelte sich im Laufe der Jahrzehnte, von der offiziellen Architekturkritik und -geschichtsschreibung zunächst nahezu unbemerkt, durch zahlreiche Eingriffe zu einem der eigenwilligsten Bauwerke des 20. Jahrhunderts. Auslöser war, wie Raynaud schreibt, die innere Notwendigkeit einer persönlichen Veränderung. "Ich musste von Neuem aufbrechen [...] mich meiner selbst wieder bemächtigen, als der, der ich war [...] Und so habe ich das Haus verschlossen, mich in 
ihm eingeschlossen. ${ }^{46}$ Diese Selbstvergewisserung mit Hilfe des Mediums der Architektur war jedoch noch nicht ausreichend, und er begann das Haus weiter zu verändern, um den verlorenen Raum, der das Substitut seiner selbst war, wiederzugewinnen. Raynaud versuchte dies über ein Material, das sich in unser kollektives Gedächtnis eingeschrieben habe, das aber auch für ihn selbst stehe: Die Oberfläche des Hauses bestand im Inneren nun mehr oder weniger vollständig aus weißen Fliesen im Standardformat $15 \times 15$ Zentimeter. Von außen so einladend wie ein Bunker, von innen so charmant wie das Pariser Leichenschauhaus, die Morgue, hatte das Haus über fünf Umbaustadien auf ihn und die Entwicklung seines künstlerischen Werks den Einfluss eines Spiegels gehabt. 1988 veröffentlichte er sämtliche Bauphasen seines Hauses und die damit verbundene Entwicklung seiner Kunst in einem ersten Buch. Nun war die architektonische Arbeit beendet, weitere Transformationen und damit seine eigene künstlerische Entwicklung schienen nicht mehr möglich. Den Künstler behinderte sein fertig gestelltes Haus, Raynaud wurde zum "prisonnier volontaire « seiner eigenen Schöpfung und damit seiner selbst. ${ }^{47}$ "Als ich 1988 begriff, dass es tatsächlich vollendet war, war das ein entsetzlicher Schock, wie das Ende einer Suche, das Ende des Lebens." Sollte er nun bis ans Ende seines eigenen Lebens der Wächter dieses 


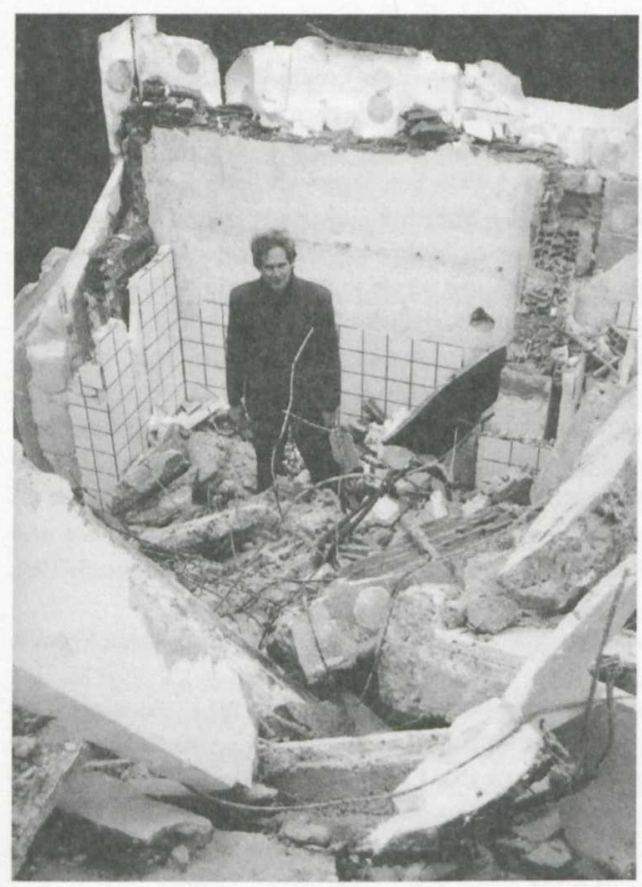

perfekten und erstarrten Kunstwerks bleiben? Die Verähnlichung mit seinem Werk und dem Haus war Raynaud nun tatsächlich zu einer Art Gefängnis geworden. Die radikale Loslösung von dem Objekt 5 Jean-Pierre Raynaud beim Abriss seines Hauses, des Hauses als Über-Ich führte Raynaud zur Zerstörung des Hauses. 22.-26. März 1993. Aus dem Abrissprozess entstand ein neues Kunstwerk, in Form einer musealen Installation, eines Films, der die Zerstörung festhält, sowie einer zweite Hausmonografie, Grabstein und Denkmal zugleich. Nicht zuletzt aber gewann Raynaud durch die 'Ermordung seines zweiten Ich seine Künstler-Identität zurück [Abb. 5]. 


\section{Endnoten}

1 Walter Benjamin, Die Mummerehlen, in: Berliner Kindheit um Neunzehnhundert. Gießener Fassung, hg. und mit einem Nachwort versehen v. Rolf Tiedemann, Frankfurt a. M. 2000, S. 7-10, hier S.7.

2 Benjamin, Mummerehlen (Anm. 1), S. 10. Schon Aristoteles hatte dieses Angleichen des Sinnesorgans mit dem Objekt der Betrachtung thematisiert, ein Gedanke, den Benjamin in dieser kurzen Erzählung vielleicht zum Anlass seiner mentstellten Welt der Kindheit" nahm. Das Buch De anima wurde unter anderem bei Diederichs in Jena 1924 neu herausgegeben: Aristoteles, Über die Seele. Ins Deutsche übertragen von Adolf Lasson. Jena 1924. Vgl.Aristoteles, Über die Seele, in: ders., Werke in deutscher Übersetzung, Buch II, Kapitel 11, 424a, Berlin 1986, S. 17f. Benjamins Text "Mummerehlen « und das in ihm dargestellte Motiv der Verähnlichung leiteten den italienischen Architekten Aldo Rossi zu eigenen Überlegungen an, die er in seiner Wissenschaftlichen Selbstbiographie von 1981 publizierte. Rossi sah sich selbst gefangen in sanalogischen Verbindungen « zwischen Dingen, Orten, Menschen und Situationen. Vgl.Aldo Rossi, Wissenschaftliche Selbstbiographie, Bern/Berlin 1991, hier vor allem S. 34 .

3 Ernst Kris und Otto Kurz, Die Legende vom Künstler. Ein geschichtlicher Versuch [1934], Frankfurt a. M. 1995.

4 Georges Rodenbach, Bruges-la-Morte [1892], Paris 1998, S. 67. Vgl. hierzu unter anderem Matthias Noell, Schönheit der Stille versus Wiederbelebung der Stadt. Georges Rodenbachs Romane Bruges-la-Morte und Le Carillonneur im Spannungsfeld von Stadtplanung und Denkmalpflege, in: Vittorio Magnago Lampugnani und Matthias Noell (Hg.), Stadtformen. Die Architektur der Stadt zwischen Imagination und Konstruktion, Zürich 2005, S. 115-129.

5 Paul Valéry, Eupalinos ou l’architecte [1921], Paris 1999, S. 28-29. Úbersetzung von Rainer Maria Rilke, Eupalinos oder der Architekt [1927], Frankfurt a. M. 1973, S. 79.

6 Alain-René Lesage, Le diable boiteux [1707]. Mit einem Vorwort v. Charles A. SainteBeuve, Paris 1874, S. 15-16; Nicolas Le Camus de Mézières, Le génie de l'architecture ou l'analogie de cet art avec nos sensations, Paris 1780. Deutsch in Auszügen übersetzt in: Von der Öbereinstimmung der Baukunst mit unseren Empfindungen, in: Allgemeines Magazin für die bürgerliche Baukunst Bd. 1, Teil 1, 1789, S. 97-172, Bd. 1, Teil 2,1790, S.66-169; Anonym, Untersuchungen über den Charakter der Gebäude; über die Verbindung der Baukunst mit den schönen Künsten, und über die Wirkungen, welche durch dieselbe hervorgebracht werden sollen, Dessau 1785, Reprint der Ausgabe Leipzig 1788, Nördlingen 1986, vgl. vor allem S. 17-18. Vgl. hierzu auch Heinz Brüggemann, Das andere Fenster: Einblicke in Häuser und Menschen. Zur Literaturgeschichte einer urbanen Wahrnehmungsform, Frankfurt a. M. 1989.

7 Anonym, Untersuchungen (Anm. 6), S. 17.

8 Johann Wolfgang von Goethe an Heinrich Meyer, 30.12.1795, in: ders., Goethes Werke (133 Bde. in 143 Teilen), hg. im Auftrage der Großherzogin Sophie von Sachsen, Weimar 1887-1919 (= Weimarer Ausgabe), hier IV, 10, S.361. Johann Wolfgang von Goethe an Major von Knebel, zit. nach Walter Dexel, Das Goethehaus in Weimar, Darmstadt 1956, S. 6. Zu den Schriftstellerhäusern vgl. auch Elisabeth Spitzbart, "Orte des Schreibens"-Anmerkungen zu Dichterhäusern des 20. Jahrhunderts und ihren geistesgeschichtlichen Voraussetzungen, in: architectura. Zeitschrift für Geschichte der Baukunst 31/1, 2001, S. 1-26; Claudia Becker, Innenwelten-Das Interieur der Dichter, in: Sabine Schulze (Hg.), Innenleben. Die Kunst des Interieurs, Ostfildern 1998, S. 170-181.

9 Johann Wolfgang von Goethe, Wilhelm Meisters Wanderjahre oder die Entsagenden. Erstes Buch, zweites Kapitel, in: ders., Goethes Werke (Anm. 8), hier I, 24, S. 16.

$10 \mathrm{John}$ Britton, The Union of architecture, sculpture and painting, exemplified by a series of illustrations, with descriptive accounts of the house and galleries of John Soane, London 1827, Vorwort S. VII. 
11 Wilhelm von Humboldt, Sonett 1091, in: Wilhelm von Humboldts Gesammelte Schriften, hg.v. d. Königlich Preußischen Akademie der Wissenschaften, Abt. 1: Werke, hg.v. Albert Leitzmann, Bd. 9: Gedichte, S. 429-430, hier S. 429. Dass ein Geist nach Ableben noch im Haus »walten « konnte, kann man jedoch bereits zuvor finden: Anonym (Von einer Dame), Vandalismus eines Weibes.-Vernichtung von Pope's Villa, in: London und Paris, 1798-1815, 20. Bd., S. 102-106.

12 Heinrich Vogeler, Brief an Hans Bethge, undatiert [Ende 1899], Deutsches Literaturarchiv Marbach a. N. Sign. 57.2163, hier zit. nach: Bernd Stenzig, Worpswede Moskau. Das Werk von Heinrich Vogeler. Worpswede 1991, S. 58.

13 Karl Scheffler, Das Haus Behrens. Mit einem Essay von Karl Scheffler, in: Dekorative Kunst, 5/9, 1901, S. 1-48, hier S. 25.

14 Marcel Proust, Auf der Suche nach der verlorenen Zeit 3. Guermantes, in: ders., Werke II, Bd.3, hg.v. Lucius Keller, Frankfurt a. M. 1996, S. 99. Jene bis zur physisch verspürten Präsenz getriebene Analogiebildung von Gegenständen, Räumen und Büchern mit einer Person findet sich auch in Thomas Bernhard, Korrektur [1975], Frankfurt a. M. 1988, S.318. Vgl. auch Prousts Kommentar zum Atelier von Gustave Moreau in Paris: Marcel Proust, Notes sur le monde mystérieux de Gustave Moreau, in: ders., Contre Sainte-Beuve, Paris 1978, S. 667-674: „[...] souvent elle n'est pas la maison d'un homme, souvent c'est-à-dire toutes les fois où il n'est plus que son âme la plus intérieure. [...] Emportez les meubles. Il ne faut plus que des toiles qui se réfêrent à l'âme intérieure où il accède souvent, et s'adressent à tous."

15 Fritz von Ostini, Villa Franz von Stuck, Darmstadt, [1909] (= Sonderdruck aus der Innen-Dekoration. Zeitschrift für Wohnungskunst und den gesamten inneren Ausbau, H. 20,1909), Zitate S. 1 u.21. Ludwig Hevesi hatte im Wiener Tagblatt, 11. November 1898, den Vergleich von passendem Haus und maßgefertigter Kleidung schon zuvor auf Victor Hortas Maison Tassel angewendet: $n E$ ist dieses das erste jener berühmten modernen Häuser [die Maison Tassel], die ihrem Eigentümer wie ein fehlerlos geschnittener Anzug Anzug passen. Es beherbergt den Mann, für den es gebaut wurde, in höchst vollkommener Weise, so vollkommen wie die Schale einer Muschel.«Zit. nach Sigfried Giedion, Raum, Zeit, Architektur. Die Entstehung einer neuen Tradition, Basel, Boston, Berlin, S. 210. Zu Schneckenhäusern vgl. auch Gaston Bachelard, Poetik des Raumes, Frankfurt a. M. 2007.

16 Frederick John Kiesler, Manifeste du corréalisme, ou les états unis de l'art plastique, in: L'Architecture d'aujourd'hui 20/2 hors série, 1949, S. 81-104, hier S. 83.

17 Alexander Koch, Das Haus eines Kunstfreundes. Haus Alexander Koch, Darmstadt 1926, S. 6.

18 Hans Schiebelhuth, Persönliche Heimgestaltung. Individuelle und typische Züge, in: Innen-Dekoration 37/1, 1926, S. 26-30, hier S. 26.

19 Heinrich Koerber, Die Psychoanalyse. Die Freudsche Lehre in ihrer Theorie und Anwendung gemeinverståndlich dargestellt. Berlin 1924, zit. in H. Lang, "Die dunkle Kammer«. Von der Zweizimmer-Wohnung der Seele, in: Innen-Dekoration 37/1, 1926, S. 55-58, hier S.55. Vgl. hierzu auch Wilhelm Michel, Weiträumigkeit und Helle. Das Wesen des neuen Wohnraums, in: Innen-Dekoration 37/8, 1926, S. 298-301.

20 Bernhard, Korrektur (Anm. 14), S. 284-285.

21 Theo van Doesburg, Architectuurvernieuwingen in het buitenland. De architectuurtentoonstelling , Die Wohnunge te Stuttgart, in: Het Bouwbedrijf 4/24,1927, S. 556-559. Deutsche Übersetzung zit. nach Theo van Doesburg, Úber europäische Architektur. Gesammelte Aufsätze aus Het Bouwbedrijf 1924-1931, Basel/Berlin/Boston 1990, S. 161-169, hier S. $165 f$.

22 Bruno Taut, Ein Wohnhaus. Mit 104 Fotos und 72 Zeichnungen, einer Farbaufnahme und einer Farbzusammenstellung, Stuttgart 1927, S. 4.

23 Hans Josef Zechlin, Taut, Bruno. Ein Wohnhaus, in: Wasmuths Monatshefte für Baukunst 11, 1927, S. 380-384.

"lch aber bin entstellt von Ähnlichkeit mit allem, was hier um mich ist." Das Motiv des architektonischen Selbstporträts in Literatur und Architektur 


\section{Endnoten}

24 Zum Aspekt des Autobiographischen im Kunstwerk vgl.u.a. Gabriele Woithe, Das Kunstwerk als Lebensgeschichte. Zur autobiographischen Dimension Bildender Kunst, Berlin 2008.

25 Zur Zeichnung Goethes vgl. Donat de Chapeaurouge, Das Milieu als Porträt, in: Wallraff-Richartz-Jahrbuch 22,1960, S.137-158, hier S.137, Abb.76. "Dans l'écriture même de la Vie de Henry Brulard, le dessin tient une place tout à fait exceptionnelle, peut-être unique dans les annales de l'autobiographie.« Béatrice Didier, La Vie de Henri Brulard ou de l'œdipe à l'écriture, in: Stendhal, Vie de Henri Brulard, Paris 1973, S.7-23, hier S. 14. Auch Xavier de Maistres zwei Zimmer-Reisen mit der Beschreibung seiner eigenen Wohnung können als solche Verschränkung von autobiografischer Schrift und eigener Architektur gelten (freundlicher Hinweis von Harald Tausch): Xavier de Maistre, Voyage autour de ma chambre, Turin 1794; ders., Expédition nocturne autour de ma chambre, Paris 1825.

26 Vgl. Stendhal, Vie de Henry Brulard (Anm. 25), S. 170. Aldo Rossi verhalf diese eigenartige Liaison von Planzeichnung und Selbstreflektion zu einer »ersten Aneignung von Architektur«, Vgl. Aldo Rossi, Selbstbiographie (Anm. 2), S. 18.

27 Werner Hofmann, Uber Menzels ,Atelierwand in der Hamburger Kunsthalle, in: Werner Hager und Norbert Knopp (Hg.), Beiträge zum Problem des Stilpluralismus, München 1977, S. 141-148, hier S. 142. An Gustave Courbets Atelier hat W. Hofmann exemplarisch eine solche Atelierszene in ihren verschiedenen Bedeutungsebenen analysiert: Das irdische Paradies. Motive und Ideen des 19. Jahrhunderts, München 1974, S.11-32 und S. 195-212. Vgl. auch Beatrice von Bismarck, Künstlerräume und Künstlerbilder. Zur Intimität des ausgestellten Ateliers, in: Schulze, Innenleben (Anm.8), S. 312-321, hier S. 312-313.

28 Erika Billeter, Malerei und Photographie im Dialog von 1840 bis heute. Mit Beiträgen von J. A. Schmoll gen. Eisenwerth, Bern 1977, hier S. 170-179.

29 Vgl.u.a. Frans Postma und Cees Broekraad (Hg.), 26, Rue du Départ. Mondrians Studio in Paris, 1921-1936, Berlin 1995. Delbo fotografierte das Atelier im März 1926, Kertész im Oktober/November 1926, vgl. ders.S. 56 u.S. 74.

30 Hofmann, Menzel (Anm.27), S. 142.

31 Zum Hut und anderen Gegenständen vgl. Michel Seuphor, Seuphor's hat. Memories of 26 Rue du Départ Paris, in: Postma/Broekraad, 26, rue du Départ (Anm. 29), S.8-17. Der stets in finanziellen Schwierigkeiten steckende Mondrian verdiente sein Geld lange Zeit mit der Ausführung von Blumenstilleben, vgl. hierzu z. B. den Brief Mondrians an Anthony Kok vom 5. Dezember 1922, zit. in: Bois, Yve-Alain, Piet Mondrian $1872-$ 1944, Bern 1995, S. 210 [Katalog zur Ausstellung "Piet Mondrian 1872-1944«, Haags Gemeentemuseum, Den Haag 1994/1995, National Gallery of Art, Washingston, D. C., The Museum of Modern Art, New York].

32 Vgl. hierzu u. a. Beatriz Colomina, Privacy and publicity. Modern architecture as mass media, Cambridge, MA, 1994, S.283-289. Diesen Hinweis verdanke ich Winfried Nerdinger.

33 Michel Frizot, Kertész, der Gärtner, in: André Kertész in Paris. Photographien 19251936, hg.v. der Collection Donations im Französischen Kulturministerium, München u.a. 1992, S. 35-43, hier S. 38. Vgl. auch Andreas Beyer, L'Atélier-c'est moi. Zu Matthias Petrus Schallers Werkbildnissen, in: Matthias Petrus Schaller (Hg.), Werkbildnis, München 2002, S.7-11 [Katalog zur Ausstellung, "Biennale d'Architettura", Venedig 2002]. Schallers »Werkbildnisse rekurrieren deutlich auf diese Ikonen der modernen Fotografie.

34 Vgl. hierzu u. a. Rolf Sachsse, Lucia Moholy-Bauhaus-Fotografin, Berlin 1995.

35 Matthias Noell, "Choisir entre l'individu et le standard. -Das Künstlerhaus bei Gropius, Le Corbusier, Van Doesburg, Bill, in: Isabelle Ewig, Thomas W. Gaehtgens u. Matthias Noell (Hg.), Das Bauhaus und Frankreich. Le Bauhaus et la France (= Passagen. Jahrbuch des Deutschen Forums für Kunstgeschichte 4), Berlin 2002, S. 83-115. 


\section{Endnoten/Abbildungsnachweis}

Zu den Schreibtischporträts im ausgehenden 19. Jahrhundert vgl. u.a. Enno Kaufhold, Bilder des Ubergangs. Zur Mediengeschichte von Fotografie und Malerei in Deutschland um 1900, Marburg 1986, S.97f.; Eva Mongi-Vollmer, Das Atelier des Malers. Die Diskurse eines Raumes in der zweiten Hälfte des 19. Jahrhunderts, Berlin 2004, S. $186 f$.

36 Le Corbusier, Maisons en série, in: ders., Vers une architecture [1923], Reprint der 3. Aufl. Paris 1928, Paris 1995, S. 185-224, hier S. 201 [Ưbers. M.N.].

37 Valéry, Eupalinos (Anm. 5), S.78.

38 François Chaslin, De l'architecture de l'atelier, in: Feuilles 7, 1984, S. 19-29, hier S. 20. Christine Hoh-Slodczyk, Das Haus des Künstlers im 19. Jahrhundert, München 1985 (= Materialien zur Kunst des 19. Jahrhunderts 33), hier S. 99.

39 Curzio Malaparte, Ritratto di pietra, abgedr. u.a. in: Marida Talamona, Casa Malaparte. Mit einem Vorwort v.Giorgio Ciucci, Mailand 1991, S.81-82, S.81. Deutsche Fassung zit. nach dies., Die Casa Malaparte und das Kap Massulo, in: Daida$\operatorname{los} 17 / 63,1997$, S. 46-55, S. 55.

$40 \mathrm{Zu}$ Briefkopf u. Zitat s. Sergio Attanasio, Curzio Malaparte. "Casa come me Punta del Massullo, tel. 160 CAPRI. Mit einem Vorwort v. Massimo Rosi, Neapel 1990, S. 24 und Anhang, sowie Petra Liebl-Osborne, „Ein Haus wie ich «. Die Casa Malaparte auf Capri, München 1999, S. 15.

41 Zu Buzzi vgl. Alberto Giorgio Cassani, La Scarzuola 1956-2004. L'autobiografia in pietra di Tomaso Buzzi, in: Casabella 68/722, 2004, S. 62-66; in ders. auch die beiden Artikel: Domenico Luciani, Requiescat, S.77; Alberto Giorgio Cassani, Migrazioni di un simbolo. Gli occhi volanti di Tomaso Buzzi, S. 79-87 sowie den Beitrag von Winfried Nerdinger im vorliegenden Band.

42 »Den är som ett självporträtt av Lind; förfinat, blekt och klassiskt klart.« L.R. [Leif Reinius], Villor, in: Byggmästaren 28/1, 1949, S. I.

43 Alfred Jericke, Goethe und sein Haus am Frauenplan, Weimar 1959, S. 19.

44 G[ustav] F[riedrich] Hartlaub, Das Selbstbildnerische in der Kunst, in: ders. u. Felix Weissenfeld, Gestalt und Gestaltung. das Kunstwerk als Selbstdarstellung des Künstlers, Krefeld 1958, S.77-135, hier S.115f.

45 Erich Steingräber, Der Bungalow. Wohn- und Empfangsgebäude für den Bundeskanzler in Bonn. Fotografiert von Paul Swiridoff, Pfullingen 1967, S. 53.

46 Jean-Pierre Raynaud, La maison 1993, Paris 1993, o. S. [Übersetzung M. N.]. Alle weiteren Zitate entstammen ebenfalls diesem kurzen Text. Vgl. hierzu Matthias Noell, Das Haus und sein Buch. Moderne Buchgestaltung im Dienst der Architekturvermittlung, Basel 2009 (= Standpunkte Dokumente 1), S. 51-55.

47 Vgl. hierzu auch Rem Koolhaas’ „Voluntary Prisoners«, Rem Koolhaas u. Bruce Mau, Small, Medium, Large, Extra-Large. Office for Metropolitan Architecture, Rotterdam 1995, S.7.

\section{Abbildungsnachweis}

1 Hugo Schmölz, Fensterplatz mit Schreibtisch im Herrenzimmer, 1925/26, aus: Alexander Koch, Das Haus eines Kunstfreundes. Haus Alexander Koch, Darmstadt 1926, S. 50.

2 Stendhal, Cabinet de M. Le Roy, um 1835/36, aus: Stendhal, Vie de Henri Brulard, Paris 1973, S. 170.

3 André Kertész, Atelier Piet Mondrians in der Rue de Départ in Paris, 1926, aus: André Kertész in Paris, München 1992, S. 56.

4 Lucia Moholy, Wohnungen der Bauhausmeister. Schreibtischklapplampe am Doppelschreibtisch des Einzelhauses Gropius, 1926, aus: Walter Gropius, Bauhausbauten Dessau, München 1930, S. 130.

5 Jean-Pierre Raynaud beim Abriss seines Hauses, 22.-26. Mărz 1993, aus: Jean-Pierre Raynaud, La maison 1993, Paris 1993, o.S. 